\title{
Sobre el origen y desarrollo del libro Semiología Médica
}

\author{
ALEJANDRO GOIC G.
}

\section{Origin and development of the book Medical Semiology}

Semiotics and Semiology share a similar etymology and meaning: the study of signs. In Medicine, signs are objective manifestations of disease, as opposed to the subjective nature of symptoms. Medical semiology comprises the study of symptoms, somatic signs and laboratory signs, history taking and physical examination (in English-speaking countries is known as Bedside diagnostic examination or Physical diagnosis). The first edition of Medical Semiology dates from 1987, and new editions appeared in 1999, 2010, and 2017. The book is devoted to semiology proper with clinical orientation. Its origin, however, dates back to 1937, when the University of Chile appointed Dr. Hernán Alessandri (1900-1981), the eminent Chilean medical educator, Professor in Semiology at the Internal Medicine Section of the Hospital del Salvador in Santiago. The authors of the present book served as Dr. Alessandri's teaching assistants for decades. The two-semester course in semiology had a tutorial character: each teaching assistant was assigned five students whom engaged daily in practical activities in the hospital wards for a total of four hours, in addition to a 45- minute lecture on the theoretical aspects of the subject. The 720-page fourth edition of the book brings together teaching method and clinical experience of more than 50 years. The book consists of six Sections: "Cardinal manifestations of disease", "Major clinical syndromes", "History taking and Physical examination", "Clinical diagnosis and the patient-physician relationship", "Laboratory clinical tests and Instrumental exploration of the body," and "Glossary of diseases." In its forty Chapters, a total of 207 issues are described in detail.

(Rev Med Chile 2018; 146: 387-390)

Key words: Education, Medical; History of Medicine; Medical History Taking; Signs and Symptoms.

L os términos Semiología y Semiótica tienen una etimología y una definición similar: el estudio de los signos. Ambos se han utilizado como sinónimos y se insertan en la Teoría general de los signos,complejo asunto del que se ocupan filósofos, lingüistas y científicos sociales. Ellos, desde diversas perspectivas, analizan los sistemas de signos o señales que comunican algo y que el hombre y los demás seres reciben de otros seres, incluyendo por cierto el lenguaje humano ${ }^{1}$. El término Semiología fue acuñado por el lingüista suizo Ferdinand de Saussure (1857-1913) y el de
Profesor Emérito, Universidad de Chile.

Miembro de Número Academia Chilena de Medicina, Instituto de Chile. Santiago, Chile.

Conflicto de intereses: Declaro no tener conflicto de intereses.

Correspondencia a: Alejandro Goic Hernando de Magallanes 1298, Las Condes, Santiago, Chile. alejandrogoicster@gmail.com
Semiótica porel filósofo, lógico y científico norteamericano Charles Sanders Pierce(1839-1914). Cada saber tiene su propio sistema de signos: semiología del derecho, semiología cultural y literaria, semiología musical, etc.

Para la Semiología médica la palabra signos tiene un significado específico y concreto: manifestaciones de enfermedad que pueden ser percibida por un observador externo (ejemplo, ictericia) en contraste con el carácter subjetivo del síntoma (ejemplo: dolor). Esta distinción, hoy familiar y evidente, sólo se consolidó en las últimas décadas 
del siglo XIX de manos del médico irlandés Richard Quain(1816-1898) y el inglés RobleyDunglison(1798-1869).

El saber médico semiológico radica en la facultad humana de la observación perspicaz, la indagación intencionada y la idea de enfermedad que la reflexión médica ha ido configurando en cada época de la historia. Si bien la Semiología Médica es una disciplina relativamente reciente, ha estado presente como un saber práctico desde el siglo $\mathrm{V}$ a.C. en el hacer de los médicos hipocráticos. Su contenido y consistencia fue paulatinamente enriquecida a través de los siglos por la observación inteligente y sagaz de diversos facultativos europeos de fines del siglo XVIII, y particularmente, del XIX y comienzos del XX. Como disciplina autónoma adquirió una identidad teórica sólida en las primeras décadas del siglo pasado.

Sabemos que la Semiología médica es el cuerpo de conocimientos que se ocupa de cómo identificar las diversas manifestaciones de enfermedad: lossintomas y sindromes manifestaciones subjetivas, los signos físicos o manifestaciones objetivas y los signos de laboratorio o exámenes complementarios. La semiología nos enseña cómo buscar, identificar y jerarquizar estas manifestaciones de enfermedad (semiotécnica); $y$, mediante el razonamiento, cómo interpretar los hallazgos semiológicos (clínica semiológica). Para la búsqueda e identificación de síntomas y signos dispone fundamentalmente de la observación, el interrogatorio del paciente (anamnesis)y el examen físico general y segmentario utilizando las técnicas clínicas de inspección, percusión, palpación y auscultación de los diversos segmentos corporales; y, eventualmente, de exámenes complementarios de laboratorio. Todo este complejo proceso requiere de parte del médico de un conocimiento acabado de las diversas entidades mórbidas o enfermedades que le permitan formular una hipótesis diagnóstica bien fundamentada. Además, debe conocer la disponibilidad de los diversos exámenes de laboratorio, sus indicaciones específicas según la hipótesis diagnóstica formulada y la interpretación de sus resultados en función de los hallazgos clínicos. Gracias al dominio y ponderación razonada de este cuerpo de conocimiento, alimentado por la experiencia clínica, el médico formula el diagnóstico.

Pero la Semiología no es sólo un saber de carácter técnico sino que está inserta en la relación del médico con su paciente: una relación interpersonal e intersubjetiva entre una persona que busca ayuda $y$, otra, que posee los conocimientos y habilidades y la disposición de ayudarla respetando su inteligencia, su dignidad y su intimidad.

Hay que señalar que los contenidos de la Semiología médica se han visto tensionados por el progreso de los conocimientos y el desarrollo tecnológico. En cierto sentido y en términos relativos, el médico contemporáneo es un profesional distinto a sus antecesores. En la década de los años 50 nuestra generación no contaba con la ecografía, la tomografía computada, la resonancia nuclear magnética o los endoscopios flexibles; sólo disponíamos de la radiología simple y con medio de contraste y de endoscopios rígidos. Esto condicionaba en la práctica clínica una exigencia semiológica relativa mayor y de un fino razonamiento clínico.

En la actualidad podemos observar una subvaloración del saber semiológico clásico y una suerte de obsesión por el uso indiscriminado de las tecnologías modernas. Con todo, en el saber médico occidental perduran las sutiles observaciones semiológicas y procedimientos clínicos descritos por los médicos a través de los siglos.

Como bien señala el académico colombiano Juan Carlos Eslava: "La semiología médica, como técnica y saber acerca de las manifestaciones de la enfermedad, sigue estando ligada de forma estrecha al conocimiento científico de la enfermedad y al dominio técnico del mundo. Esto no ha cambiado, a pesar de que el ropaje del saber semiológico adopte las lógicas propias del lenguaje computacional y de los enunciados probabilísticos"2.

Aun cuando la primera edición de nuestro libro Semiología Médica se publicó en 1987, hace 30 años, sus raíces se remontan a 1931 cuando el Profesor Dr. Hernán Alessandri Rodríguez se incorporó al Hospital del Salvador de Santiago. Fue nombrado por la Universidad de Chile, primero, como Profesor de la Cátedra Extraordinario de Patología médica en 1932 y, luego, en 1937, Profesor de Semiología médica. Se unieron a él como sus ayudantes jóvenes médicos internistas que, bajo su inspiración, se dedicaron a la asistencia médica y la docencia y fueron protagonistas del incipiente desarrollo en el país de las especialidades derivadas de la Medicina Interna; entre ellos el Dr. Gastón Chamorro Zapata en 1934. En las décadas siguientes, la Cátedra y Servicio de Medicina del Hospital del Salvador alcanzó un gran desarrollo 
y prestigio, convirtiéndose en el modelo docente-asistencial más ejemplar que personalmente hayamos conocido; estudiantes de pregrado, Internos y Residentes-becarios se sentían orgullosos de formarse allí. Era un Servicio clínico de gran disciplina médica del equipo de salud, entendida como el cumplimiento riguroso y oportuno de las tareas asistenciales y docentes que se les asignaba fuesen funcionarios del Servicio Nacional de Salud (SNS) o de la Universidad de Chile. La atención profesional médica y de enfermería proporcionada en este Servicio de Medicina comprendía las $24 \mathrm{~h}$ del día, incluyendo fines de semana y festivos, con presencia de un médico Residente- becario de turno y de una auxiliar de enfermería. Técnicamente hablando, este servicio clínico era superior en su organización y calidad de atención que cualquier clínica privada de esa época. En su estructura $\mathrm{y}$ funcionamiento tuvieron un rol significativo colaboradores cercanos al Profesor Alessandri: los Doctores Héctor Ducci Claro, poseedor de cualidades administrativas excepcionales y Eliseo Concha Parot, un excelente internista y docente metódico y estricto.

La Cátedra de Alessandri dio especial importancia a la enseñanza de la Semiología Médica. La asignatura cubría 2 semestres en el $3^{\text {er }}$ año de la carrera: cuatro horas diarias de actividad práctica en las salas de hospital, de carácter tutorial: 1 docente con 4 o 5 alumnos; y, luego, $45 \mathrm{~min}$ adicionales de exposiciones teóricas.

La experiencia en docencia fue construyendo un esquema ordenado y explícito para abordar los componentes esenciales de un historial clínico y del examen físico general y segmentario del paciente. Los estudiantes llevaban una ficha-docente paralela a la ficha hospitalaria, examinaban independientemente a los enfermos y sus escritos sobre la historia clínica eran discutidos grupalmente con el tutor. La ficha clínicas incluía una hoja especial destinada al Diagnóstico provisorio y sus fundamentos que debía consignarse al ingreso del paciente al hospital; esto permitía un acceso inmediato al diagnóstico en caso de una emergencia o de una interconsulta y ponía en juego el razonamiento clínico, la capacidad de síntesis del médico y su habilidad en el ejercicio del diagnóstico diferencial. Agréguese como actividad docente significativa la participación de los alumnos y médicos en las reuniones clínicas y anátomo-clínicas semanales presididas por el Profesor Alessandri, instrumentos invaluables de educación médica continua de carácter colectivo.

Cultivé una cercana amistad con el Dr. Gastón Chamorro Zapata, distinguido internista, cardiólogo clínico y semiólogo excepcional, además de excelente persona. A comienzos de los años 80 conversábamos sobre la formación de los estudiantes. Le dije: don Gastón usted ha enseñado la Semiología durante cincuenta años y yo durante veinticinco. Sería lamentable que toda la experiencia acumulada por décadas en esta Cátedra se perdiera, desvaneciéndose en el transcurso inevitable de los años. Le propongo que escribamos un texto de Semiología médica que recoja la metodología docente utilizada en la Cátedra, surgida no de la teoría sino de la práctica clínica cotidiana y la experiencia educacional. Algo dubitativo al comienzo, accedió y nos pusimos a diseñar el texto. Definimos que sería un libro propiamente semiológico con orientación clínica que contuviera los conocimientos fundamentales de la disciplina en un nivel equidistante de los tradicionales y esquemáticos "apuntes" y de los exuberantes tratados. Habíamos aprendido que inicialmente a los estudiantes les era difícil la descripción de la anamnesis próxima, lo que era comprensible porque desconocían la nomenclatura semiológica, las características de los síntomas y los signos físicos, el modo de investigarlos e interpretarlos y sus principales causas, insumos necesarios para poder construir una historia clínica coherente. Por esta razón, la estructura del libro contempló, primero, la descripción semiológica detallada de los síntomas y síndromes más comunes y, luego, la anamnesis, examen físico y los exámenes de laboratorio, en esa secuencia.

En la época no contábamos con computadores ni facilidades electrónicas de información, ni de tiempo académico libre para realizar la tarea. Nos repartimos los temas a escribir-algunos lo hicimos en conjunto- y los revisábamos críticamente. Invitamos a participar en esta aventura a destacados especialistas y académicos de las diversas Cátedras universitarias establecidas en el Hospital del Salvador. La preparación del libro nos tomó alrededor de cinco años, siendo prolongada por el eminente Dr. Amador Neghme Rodríguez (1912-1987), en aquel tiempo Presidente de la Academia Chilena de Medicina. La Editorial Mediterráneo acogió nuestra propuesta para la impresión y distribución del libro. La primera edición, de 456 páginas y 22 
colaboradores vio la luz en $1987^{3}$. La apreciada decisión de la Organización Panamericana de la Salud de incorporar el libro en su Programa de Textos fue extremadamente valiosa para su difusión en las Escuelas de Medicina nacionales y de Latinoamérica.

Con el lamentable fallecimiento del Dr. Chamorro, desde la segunda edición se incorporó como asociado el Dr. Humberto Reyes quien ha cumplido una excelente labor editorial, particularmente en la sección relativa a los exámenes de laboratorio bioquímico y a los métodos instrumentales de exploración clínica.

Las anteriores ediciones de Semiología Médica fueron publicadas en 1987, 1999 y 2010 . Tal vez, una característica singular de nuestro libro es la inclusión de temas que no suelen contener los textos semiológicos: la relación médico-paciente, semiología de la nerviosidad y ansiedad, la hostilidad en clínica, los síntomas y signos funcionales, el diagnóstico y diagnóstico diferencial, la apreciación de la gravedad de un enfermo, el diagnóstico de muerte, particularidades de la anamnesis y examen físico en el niño y en el adulto mayor, el Juramento hipocrático y sus valores éticos. Nuestra Semiología Médica está inspirada en una medicina de la persona cuyos sufrimientos no son sólo físicos sino que también psicológicos y emocionales.

Hoy presentamos la Cuarta Edición 2017 cuidadosamente revisada y ampliada con la descripción de algunos temas no presentes en las ediciones anteriores ${ }^{4}$. Todos los capítulos han sido revisados críticamente por sus autores, perfeccionándolos y actualizándolos. Esta edición incluye nuevas materias complementarias de la formación semiológica y clínica de los estudiantes entre ellas la Semiología de las hemorragias intra-craneanas e infarto cerebral, semiología de las demencias, semiología del embolismo pulmonar, semiología del prolapso genital, del parto y del puerperio, el síndrome visual por uso del computador. La diagramación es moderna y atractiva y se aumen- taron significativamente el número de tablas y de imágenes en colores ilustrando cada capítulo del texto. En el libro de 727 páginas participan 51 especialistas La preparación del texto requirió tres años de trabajo y fue impreso en Eslovenia. Comprende seis Partes o Secciones: Manifestaciones cardinales de enfermedad, Principales síndromes clínicos, Anamnesis y Examen físico, Diagnóstico clínico y Relación médico-paciente, Exámenes de Laboratorio clínico y de Exploración especializada y Glosarios de Enfermedades. En 40 capítulos se describen detalladamente 207 temas semiológicos.

Estamos complacidos por haber acompañado a generaciones de estudiantes en el aprendizaje de una disciplina que nos parece fundamental en la formación del médico. Nos hace pleno sentido la sentencia del médico francés del siglo XIX Dr. Charles Laubry(1872-1969): "La Semiología no es sólo la gramática de la medicina sino que la medicina misma”. Confiamos que esta Cuarta Edición de Semiología médica que, en su conjunto reúne conocimientos y experiencias acumuladas por décadas de médicos nacionales de gran idoneidad, siga siendo una compañía educacional efectiva para las actuales y furas generaciones de estudiantes.

\section{Referencias}

1. Morris, Charles. Fundamentos de la teoría de los signos. Ediciones Paidós: Barcelona. 1985.

2. Eslava Castañeda, JC. Semiología médica: Un breve recorrido por su historia. En: Eslava JH et al. Semiología quirúrgica. Parte I. Generalidades. Universidad Nacional de Colombia: Bogotá. 2014. páginas 19-34.

3. Goic A, Chamorro G. Semiología Médica. Editorial Mediterráneo: Santiago de Chile. 1987.

4. Neghme, A. Prefacio. En: Goic A, Chamorro, G. Semiologías Médica. Editorial Mediterráneo: Santiago de Chile. 1987.

5. Goic A, Chamorro G, Reyes H. Semiología Médica. IV edición. Editorial Mediterráneo: Santiago de Chile. 2017. 